\title{
Diboson resonant production in non-custodial composite Higgs models
}

\section{A. Carmona, ${ }^{a}$ A. Delgado, ${ }^{b, c}$ M. Quirós ${ }^{d}$ and J. Santiago ${ }^{e}$}

${ }^{a}$ Institute for Theoretical Physics, ETH Zurich, Zurich, 8093 Switzerland

${ }^{b}$ Department of Physics, University of Notre Dame, Notre Dame, IN, 46556 U.S.A.

${ }^{c}$ Theory Division, Physics Department, CERN, Geneva 23, CH-1211 Swizterland

${ }^{d}$ Institució Catalana de Recerca i Estudis Avançats (ICREA) and IFAE-UAB, Bellaterra, Barcelona, 08193 Spain

e Departamento de Fúsica Teórica y del Cosmos and CAFPE, Universidad de Granada, Campus de Fuentenueva, Granada, E-18071 Spain

E-mail: carmona@itp.phys.ethz.ch, antonio.delgado@nd.edu, quiros@ifae.es, jsantiago@ugr.es

ABSTRACT: We show that the recently reported excess in resonant diboson production can be explained in the context of non-custodial composite Higgs models. Dibosons are generated via the s-channel exchange of massive vector bosons present in these models. We discuss the compatibility of the signal excess with other diboson experimental searches. We also discuss the tension between diboson production and other experimental tests of the model that include electroweak precision data, dilepton, dijet and top pair production and show that there is a region of parameter space in which they are all compatible with the excess.

KeYwords: Phenomenological Models

ArXiv EPrint: 1507.01914 


\section{Contents}

1 Introduction 1

2 Experimental status $\quad 2$

3 Non-custodial composite Higgs models 4

4 Results $\quad 8$

5 Conclusions 11

\section{Introduction}

The ATLAS collaboration has recently reported an intriguing excess in hadronically decaying diboson $(W W / W Z / Z Z)$ resonant production peaked around $\sim 2 \mathrm{TeV}$ [1]. The observed excess corresponds to significances of $3.4 \sigma, 2.6 \sigma$ and $2.9 \sigma$ for the $W Z, W W$ and $Z Z$ channels respectively. Hadronically decaying $Z$ and $W$ are not easy to distinguish so the three channels are not exclusive. As a cuantitative example, for a $W^{\prime}$ with a $2 \mathrm{TeV}$ mass decaying to $W Z$, we have the following 95\% C.L. expected (exp) and observed (obs) limits

$$
\sigma\left(p p \rightarrow W^{\prime} \rightarrow W Z\right)<12 \mathrm{fb}(\exp ), \quad \sigma\left(p p \rightarrow W^{\prime} \rightarrow W Z\right)<36 \mathrm{fb}(\mathrm{obs}) .
$$

Although the excess is not statistical significant yet, it is interesting to entertain the possibility that it corresponds to a real signal of new physics. There is a similar search for hadronically decaying diboson production in CMS [2]. Interestingly enough there is a small excess localized near the $2 \mathrm{TeV}$ region. For a $W^{\prime}$ with a $2 \mathrm{TeV}$ mass decaying to $W Z \mathrm{CMS}$ finds

$$
\sigma\left(p p \rightarrow W^{\prime} \rightarrow W Z\right)<8 \mathrm{fb}(\exp ), \quad \sigma\left(p p \rightarrow W^{\prime} \rightarrow W Z\right)<13 \mathrm{fb}(\text { obs }) .
$$

An interpretation of these excesses in a number of models has been recently presented in [3-15]. In this article we will show that they can be explained in the context of noncustodial composite Higgs models without conflicting with any other current experimental bound.

A natural prediction of composite Higgs models is the presence of composite vector resonances that mix with the Standard Model (SM) electroweak bosons. They typically have a large coupling to the longitudinal components of the $W$ and $Z$ bosons and therefore their decay into pairs of SM gauge bosons is usually sizeable. Thus, they are prime candidates to explain an excess in diboson resonant production. The mass scale of the diboson excess $M \sim 2 \mathrm{TeV}$ is however smaller than the typical masses of the vector resonances in composite Higgs models, which, due to a sizeable tree-level contribution to the 
$S$ parameter, are expected to be in the $M \gtrsim 2.5-3 \mathrm{TeV}$ region [16-19]. However, using a soft-wall construction of higher-dimensional holographic duals of composite Higgs models it has been shown that lighter vector resonances are compatible with electroweak precision tests (EWPT) both in custodial [20] and non-custodial [21, 22] set-ups. In practice this is done by reducing the mixing between the heavy vector resonances and the SM gauge bosons and the coupling between the former and the SM fermions. Reducing these mixings and couplings allows for lighter resonances compatible with EWPT but it also reduces their production cross section and their decay branching ratio into SM vector bosons, making their collider phenomenology more challenging [23, 24]. Hence there is, in principle, a tension in these models between the constraints from EWPT and the possible size of anomalous diboson production. For the sake of simplicity we will consider in this article the minimal non-custodial composite Higgs model as a benchmark and study in detail the compatibility of the observed excess with constraints from EWPT. ${ }^{1}$

Before explaining the excesses in the context of specific models, however, one should check whether these excesses are compatible with the results of other diboson resonant searches or if, on the contrary, they are already excluded by the latter. We will show in the next section that, although there is a large number of related searches for diboson resonance production with no significant excess, the corresponding bounds are compatible with the number of events needed to explain the observed excess. Of course with a specific model in mind there are also other experimental searches that play a relevant role in constraining the parameters of the model. In particular the most important ones for non-custodial composite Higgs models are dijet, dilepton and top pair production. We will show that there are regions of parameter space in which the observed excess can be explained without contradicting any of the existing limits.

The rest of the paper is organized as follows. In section 2 we will summarize the different experimental searches for exotic resonances. In section 3 the non-custodial model will be presented including the EW fit. The results are presented in section 4 and finally section 5 is devoted to our conclusions.

\section{Experimental status}

As mentioned above, before trying to explain the observed excess in terms of a specific model, we have to make sure that the excess is not already ruled out by other related resonant searches. There are a number of other diboson (WW/WZ/ZZ) resonant searches performed by ATLAS and CMS in the semi-leptonic and purely leptonic channels. No significant excesses are observed in these searches and the resulting 95\% C.L. bounds are reported in the first block of table 1 .

At first sight it might seem that these bounds rule out the reported excess by ATLAS (and to a lesser extent CMS) in eqs. (1.1) and (1.2). However, a more detailed look at the results in ref. [1] shows that the cross section needed to explain the ATLAS excess

\footnotetext{
${ }^{1}$ Custodial models have a similar phenomenology and, although the quantitative details will differ, the general features that the diboson excess can be explained without conflict with any other experimental constraint is likely to hold also in these models.
} 


\begin{tabular}{|lcc|c|c|c|c|}
\hline \multicolumn{3}{|c|}{ Channel } & Process & $1.8 \mathrm{TeV}$ & $1.9 \mathrm{TeV}$ & $2.0 \mathrm{TeV}$ \\
\hline ATLAS & $\ell \nu j j$ & {$[25]$} & $p p \rightarrow W^{\prime} \rightarrow W Z$ & $13 \mathrm{fb}$ & $12 \mathrm{fb}$ & $10 \mathrm{fb}$ \\
CMS & $\ell \nu j j$ & {$[26]$} & $p p \rightarrow G^{*} \rightarrow W W$ & $6 \mathrm{fb}$ & $4 \mathrm{fb}$ & $3 \mathrm{fb}$ \\
ATLAS & $\ell \ell j j$ & {$[27]$} & $p p \rightarrow W^{\prime} \rightarrow W Z$ & $14 \mathrm{fb}$ & $20 \mathrm{fb}$ & $20 \mathrm{fb}$ \\
ATLAS & $\ell \ell j j$ & {$[27]$} & $p p \rightarrow G^{*} \rightarrow Z Z$ & $6 \mathrm{fb}$ & $7 \mathrm{fb}$ & $7 \mathrm{fb}$ \\
CMS & $\ell \ell j j$ & {$[26]$} & $p p \rightarrow G^{*} \rightarrow Z Z$ & $14 \mathrm{fb}$ & $12 \mathrm{fb}$ & $8 \mathrm{fb}$ \\
ATLAS & $3 \ell \nu$ & {$[28]$} & $p p \rightarrow W^{\prime} \rightarrow W Z$ & $21 \mathrm{fb}$ & $22 \mathrm{fb}$ & $21 \mathrm{fb}$ \\
CMS & $3 \ell \nu$ & {$[29]$} & $p p \rightarrow W^{\prime} \rightarrow W Z$ & $27 \mathrm{fb}$ & $20 \mathrm{fb}$ & $20 \mathrm{fb}$ \\
\hline ATLAS & $Z H$ & {$[30]$} & $p p \rightarrow Z^{\prime} \rightarrow Z H$ & $14 \mathrm{fb}$ & $16 \mathrm{fb}$ & - \\
ATLAS & $W H$ & {$[30]$} & $p p \rightarrow W^{\prime} \rightarrow W H$ & $31 \mathrm{fb}$ & $37 \mathrm{fb}$ & - \\
CMS & $Z H$ & {$[31]$} & $p p \rightarrow Z^{\prime} \rightarrow Z H$ & $13 \mathrm{fb}$ & $9 \mathrm{fb}$ & $7 \mathrm{fb}$ \\
CMS & $W H$ & {$[31]$} & $p p \rightarrow W^{\prime} \rightarrow W H$ & $14 \mathrm{fb}$ & $9 \mathrm{fb}$ & $7 \mathrm{fb}$ \\
\hline ATLAS & $\ell \ell$ & {$[32]$} & $p p \rightarrow Z^{\prime} \rightarrow \ell \ell$ & $0.23 \mathrm{fb}$ & $0.22 \mathrm{fb}$ & $0.20 \mathrm{fb}$ \\
ATLAS & $\ell \nu$ & {$[33]$} & $p p \rightarrow W^{\prime} \rightarrow \ell \nu$ & $0.54 \mathrm{fb}$ & $0.48 \mathrm{fb}$ & $0.44 \mathrm{fb}$ \\
CMS & $\ell \ell$ & {$[34]$} & $p p \rightarrow Z^{\prime} \rightarrow \ell \ell$ & $0.24 \mathrm{fb}$ & $0.24 \mathrm{fb}$ & $0.24 \mathrm{fb}$ \\
CMS & $\ell \nu$ & {$[35]$} & $p p \rightarrow W^{\prime} \rightarrow \ell \nu$ & $0.40 \mathrm{fb}$ & $0.34 \mathrm{fb}$ & $0.30 \mathrm{fb}$ \\
ATLAS & $t \bar{t}$ & {$[36]$} & $p p \rightarrow Z^{\prime} \rightarrow t \bar{t}$ & $64 \mathrm{fb}$ & $60 \mathrm{fb}$ & $52 \mathrm{fb}$ \\
CMS & $t \bar{t}$ & {$[37]$} & $p p \rightarrow Z^{\prime} \rightarrow t \bar{t}$ & $17 \mathrm{fb}$ & $14 \mathrm{fb}$ & $11 \mathrm{fb}$ \\
ATLAS & dijets & {$[38]$} & $p p \rightarrow W^{\prime} \rightarrow j j$ & $270 \mathrm{fb}$ & $184 \mathrm{fb}$ & $119 \mathrm{fb}$ \\
CMS & dijets & {$[39]$} & $p p \rightarrow W^{\prime} \rightarrow j j$ & $205 \mathrm{fb}$ & $155 \mathrm{fb}$ & $95 \mathrm{fb}$ \\
\hline
\end{tabular}

Table 1. Summary of the relevant 95\% C.L. observed bounds.

is in principle compatible with all these extra constraints. The most significant excess is observed in the two $100 \mathrm{GeV}$ bins with invariant mass $1850 \mathrm{GeV} \leq m_{V V^{\prime}} \leq 2050 \mathrm{GeV}$ and it corresponds to 8, 6.5 and 6.5 events over the expected background for the $W Z, W W$ and $Z Z$ selection regions, respectively [40]. Assuming that the diboson resonance corresponds to a vector boson exchanged in the s-channel, the total number of events can be computed as follows

$$
N_{\mathrm{ev}}=\mathcal{L} \times \epsilon \times \sigma\left(p p \rightarrow V^{\prime}\right) \times B R\left(V^{\prime} \rightarrow V_{1} V_{2}\right) \times B R\left(V_{1} \rightarrow j j\right) \times B R\left(V_{2} \rightarrow j j\right),
$$

where $\mathcal{L}=20.3 \mathrm{fb}^{-1}$ is the integrated luminosity, $V^{\prime}$ is the intermediate vector boson, $V_{1,2}$ stand for a $W$ or $Z$ boson and $\epsilon$ is the analysis efficiency. From table 1 and figure 2(b) of ref. [1] we conservatively estimate

$$
\epsilon \approx 0.14 \times 0.7 \approx 0.1
$$

where 0.7 is the fraction of events in the region around the $V^{\prime}$ mass. Inserting the numbers in eq. (2.1) we obtain

$$
\sigma\left(p p \rightarrow V^{\prime}\right) \times B R\left(V^{\prime} \rightarrow V_{1} V_{2}\right) \approx N_{\mathrm{ev}} \mathrm{fb} \approx 6-8 \mathrm{fb},
$$

where we have assumed similar efficiencies for the reconstruction of the hadronic $Z$ and $W$ and for simplicity we have neglected the small difference in their hadronic branching 
fractions. A proper statistical combination of all three different channels has not been provided by the ATLAS collaboration but they emphasize that a number of events will populate several regions. Instead of trying to estimate the contamination across channels we will simply add the cross sections in all of them. This is a reasonable approximation, taking into account that we have been conservative when estimating the efficiencies. Thus, we see that there is no obvious contradiction between the required cross section to explain the observed ATLAS excess with the limits from other searches. The only possible exception is the CMS $\ell \nu j j$ analysis [26], which puts a constraint on $\sigma\left(p p \rightarrow G^{*} \rightarrow W W\right) \leq 6-3 \mathrm{fb}$ for $M=1.8-2 \mathrm{TeV}$. As we will discuss below, our model does not give a contribution to this signal with the same strength as to the fully hadronic one. Furthermore, the interpretation of the bound in terms of a spin- 2 resonance makes it difficult to apply it to our model without further assumptions.

We finally show in the second and third blocks of table 1 other experimental constraints that will be relevant for the non-custodial composite Higgs model that we will use to explain the diboson excess. Searches for exotic resonances decaying into a $\mathrm{W}$ or $\mathrm{Z}$ boson and a Higgs boson have been performed by ATLAS and CMS. No significant excess is found in these channels and the $95 \%$ C.L. bounds on cross-sections are summarized in the second block of table 1 . Finally other resonant channels, as $\ell \ell, \ell \nu, t \bar{t}$ and dijets have been searched for by ATLAS and CMS and the resulting $95 \%$ bounds on cross-sections are summarized in the third block of table 1 . We will include all the bounds from the second and third blocks of table 1 in our numerical analysis below.

\section{Non-custodial composite Higgs models}

Composite Higgs models provide an elegant solution to the hierarchy problem. In these models the Higgs boson is a composite resonance of a new strongly coupled interaction and its mass is protected by its finite size. Further assuming that the Higgs is a pseudo-NambuGoldstone boson of a global symmetry of the strongly interacting sector ensures that its mass is naturally much smaller than the composite scale [41-43]. A common prediction of these models is the presence of electroweak composite vector resonances with masses around or above the scale of compositeness and a large mixing with the SM gauge bosons. Due to a sizeable tree-level contribution to the $S$ parameter, these vector resonances are however expected to be in the $M \gtrsim 2.5-3 \mathrm{TeV}$ region, even in custodially symmetric set-ups [16-19]. Using a soft-wall construction of higher-dimensional holographic duals of composite Higgs models it has been shown that lighter vector resonances are compatible with EWPT both in custodial [20] and non-custodial [21, 22] models. Indeed, vector resonances as light as $1 \mathrm{TeV}$ are allowed without violating the very stringent constraints from EWPT. In practice this is done by effectively reducing the mixing between the composite vector resonances and the SM gauge fields. The $T$ parameter, which is volume enhanced in these models, is proportional to the square of this mixing whereas the $S$ parameter, which does not have the volume enhancement and is therefore naturally smaller than the $T$ parameter, is linearly proportional to the mixing. Thus, the reduction in the mixing naturally brings the model back in the allowed ellipse in the $S-T$ plane with much lighter vector resonances. In the 
explicit construction used in $[21,22]$ the coupling of the composite vectors to the light SM fermions is also reduced with respect to models in $\mathrm{AdS}_{5}$ thus reducing the production cross section of the vector resonances and making their collider phenomenology more difficult to explore $[23,24]$. The reduced mixing between the composite and SM vectors also has a direct implication relevant for this work. This mixing governs the decay of the massive vector resonances into pairs of SM gauge bosons and therefore reducing it to ease the constraints from EWPT also reduces the potential signal to explain the diboson excess.

In order to be quantitative and explore a region of parameter space as large as possible in this class of models without the burden of constructing complete non-trivial gravitational backgrounds, we consider a simplified version of the non-custodial composite Higgs model in which we include the minimal number of fields that are relevant for the diboson production and for the associated constraints. Specifically we consider only the first resonances with the quantum numbers of the electroweak SM gauge bosons. Color octet vector and fermionic resonances are also typically present in these models but their features are model dependent and would unnecesarily complicate the current analysis. Furthermore there are stringent constraints from flavor, dijet and top production on color octet vector resonances [44-47] that are therefore expected to be heavier than the needed $\sim 2 \mathrm{TeV}$ to explain the diboson excess. $^{2}$

The relevant part of the Lagrangian reads

$$
\mathcal{L}=\mathcal{L}_{\text {SM }}^{(0)}+\mathcal{L}_{\text {Gauge }}^{(1)}+\mathcal{L}_{\text {Fermion }}^{(1)}+\mathcal{L}_{\text {Higgs }}^{(1)},
$$

with

$$
\begin{aligned}
& \mathcal{L}_{\text {Gauge }}^{(1)}=-\frac{1}{4} W_{\mu \nu}^{(1) i} W^{(1) i \mu \nu}-\frac{1}{4} B_{\mu \nu}^{(1)} B^{(1) \mu \nu}+\frac{1}{2} M^{2} W_{\mu}^{(1) i} W^{(1) i \mu}+\frac{1}{2} M^{2} B_{\mu}^{(1)} B^{(1) \mu} \\
& -g \varepsilon^{i j k} \partial_{[\mu} W_{\nu]}^{(1) i} W_{\mu}^{(1) j} W_{\nu}^{(0) k}-\frac{1}{2} g \varepsilon^{i j k} \partial_{[\mu} W_{\nu]}^{(0) i} W_{\mu}^{(1) j} W_{\nu}^{(1) k}, \\
& \mathcal{L}_{\text {Higgs }}^{(1)}=\frac{g g_{V}}{2} W_{\mu}^{(1) i} \phi^{\dagger} \mathrm{i} \stackrel{\leftrightarrow}{D}^{i \mu} \phi+\frac{g^{\prime} g_{V}}{2} B_{\mu}^{(1)} \phi^{\dagger} \mathrm{i} \stackrel{\leftrightarrow}{D}^{\mu} \phi, \\
& \mathcal{L}_{\text {Fermion }}^{(1)}=\sum_{\psi_{L}} g g_{\psi_{L}} W_{\mu}^{(1) i} \bar{\psi}_{L} \gamma^{\mu} \frac{\sigma^{i}}{2} \psi_{L}+\sum_{\psi} g^{\prime} g_{\psi} Y_{\psi} B_{\mu}^{(1)} \bar{\psi} \gamma^{\mu} \psi
\end{aligned}
$$

where $\stackrel{\leftrightarrow}{D}^{i \mu} \equiv \sigma^{i} D^{\mu}-\overleftarrow{\leftarrow}^{\mu} \sigma^{i}, \stackrel{\leftrightarrow}{D}^{\mu} \equiv D^{\mu}-\overleftarrow{\leftarrow}^{\mu}, Y_{\psi}$ stands for the hypercharge of fermion $\psi$ and $\psi_{L}$ and $\psi$ run over all left-handed SM fermions and all SM fermions, respectively. $D^{\mu}$ stands for the SM covariant derivative and the square brackets denote antisymmetrization with respect to the indices. We assume that all quarks, except for the right-handed top and the left-handed top-bottom doublet, have the same coupling, while all leptons share the same coupling, which can be different in general from the one of quarks. ${ }^{3}$ Thus we have

\footnotetext{
${ }^{2}$ As we will see below, our choice of parameters is consistent with the flavor symmetry proposed in [48], which significantly relaxes the flavor constraints. These are further reduced when only electroweak vector resonances are light as we assume.

${ }^{3}$ In composite Higgs models this can be naturally explained due to the different mass generation mechanism between quarks and leptons [49-52].
} 
five relevant parameters that control the mixing between the composite vectors and the SM gauge bosons $\left(g_{V}\right)$ and the couplings of the composite vectors to the SM leptons $\left(g_{1}\right)$, light quarks $\left(g_{q}\right)$, left-handed top and bottom quarks $\left(g_{q_{3}}\right)$ and right-handed top quark $\left(g_{t_{\mathrm{R}}}\right)$, respectively (the mass of the massive vectors is essentially fixed by the scale of the ATLAS excess). All these parameters but $g_{t_{\mathrm{R}}}$ enter the contributions to EWPT. $g_{q}$ controls the production cross section of the composite resonances and $g_{V}$ their decay into pairs of SM gauge bosons. $g_{t_{\mathrm{R}}}$ has a global impact on the decays of the composite vectors because it is expected to be sizeable (from the top mass) and therefore can give a large contribution to the total width of the composite vectors.

The Lagrangian (3.3) contains mixing terms between the composite $Z$ and $W^{ \pm}$resonances and their SM counterparts, leading to the following mass terms

$$
\mathcal{L} \supset \frac{1}{2}\left(Z_{\mu}^{(0)} Z_{\mu}^{(1)}\right) \mathcal{M}_{Z}^{2}\left(\begin{array}{c}
Z_{\mu}^{(0)} \\
Z_{\mu}^{(1)}
\end{array}\right)+\left(W_{\mu}^{(0)+} W_{\mu}^{(1)+}\right) \mathcal{M}_{W}^{2}\left(\begin{array}{l}
W_{\mu}^{(0)-} \\
W_{\mu}^{(1)-}
\end{array}\right),
$$

with

$$
\mathcal{M}_{X}^{2} \approx\left(\begin{array}{cc}
m_{X}^{2} & g_{V} m_{X}^{2} \\
g_{V} m_{X}^{2} & M^{2}
\end{array}\right), \quad \text { for } \quad X=Z, W
$$

where $W_{\mu}^{(1) \pm}$ and $Z_{\mu}^{(1)}$ (and $A_{\mu}^{(1)}$ ) are defined exactly as their SM counterparts. Even though there are no interaction terms in (3.2) between one heavy resonance and two light states (reminiscent of the orthonormality of the different KK modes in the holographic duals), the diagonalization of (3.5) leads to such couplings when the required rotation is implemented in the $V_{1}^{(0)} V_{2}^{(0)} V_{3}^{(0)}$ and the $V_{1}^{(1)} V_{2}^{(1)} V_{3}^{(0)}$ couplings. In particular, after going to the physical basis $\left(Z_{\mu}, Z_{\mu}^{\prime}\right)$ and $\left(W_{\mu}^{ \pm}, W_{\mu}^{\prime \pm}\right)$, one gets $\mathcal{O}\left(m_{W}^{2} / M^{2}\right)$ suppressed couplings $Z^{\prime} W^{+} W^{-}, W^{\prime \pm} W^{\mp} Z$ and $A^{\prime} W^{+} W^{-}$(where $A^{\prime}$ is just $A^{(1)}$, since the photon resonance does not mix with the zero-mode). Note that in the unitary gauge that we are working on the couplings of the heavy vector resonances to the SM gauge bosons are suppressed. Nevertheless the enhanced decay into the longitudinal components of the SM gauge bosons provides the sizeable branching ratio that we would expect from the couplings to the electroweak Goldstone bosons in a renormalizable gauge. On the other hand, since the couplings $Z^{(1)} Z^{(0)} H$ and $W^{(1) \pm} W^{(0) \mp} H$ are already present in (3.3), the effect of the aforementioned rotation is subleading and we can safely neglect it for the present phenomenological study.

In our model SM diboson resonances proceed through the following processes

$$
\begin{aligned}
q \bar{q}^{\prime} & \rightarrow W^{\prime \pm} \rightarrow W^{ \pm} Z, \\
q \bar{q} & \rightarrow Z^{\prime} / A^{\prime} \rightarrow W^{+} W^{-} .
\end{aligned}
$$

We therefore have no anomalous $Z Z$ production and will have to account for the observed excess with the $W W$ and $W Z$ samples. In the fully hadronic case we will neglect the small differences in the reconstruction of the hadronic $W$ and $Z$ and therefore we will simply add the $W W$ and $W Z$ production cross sections in order to estimate the signal excess. These 
processes are accompanied by the corresponding ones with the Higgs boson

$$
\begin{aligned}
q \bar{q}^{\prime} & \rightarrow W^{\prime \pm} \rightarrow W^{ \pm} H, \\
q \bar{q} & \rightarrow Z^{\prime} \rightarrow Z H .
\end{aligned}
$$

As we have indicated, the heavy vector boson production is controlled by the coupling to light quarks $g_{q}$. Their decay into dibosons (including the Higgs boson) is proportional to $g_{V}$ but it is also sensitive to all the other couplings through the vectors' widths. They can also decay in any of the other possible channels, namely into dijets (through $g_{q}$ ), tops (through $g_{q_{3}}, g_{t_{\mathrm{R}}}$ ) or dileptons (through $g_{1}$ ). As we have discussed in section 2, all these channels have quite stringent constraints on the production cross section of new resonances with a $\sim 2 \mathrm{TeV}$ mass. Regarding the very stringent bound from the CMS $\ell \nu j j$ search [26], our model contributes to it through both $W W$ and $W Z$ production. Nevertheless, the $W Z$ channel contributes with a factor $1 / 2$ with respect to the $W W$ one, due to the multiplicity of the latter in the semi-leptonic decay. Thus, neglecting the differences in efficiencies (and hadronic decays) in the hadronic $W$ and $Z$ reconstructions we have

$$
\sigma\left(p p \rightarrow Z^{\prime} / A^{\prime} \rightarrow W W\right)+\frac{1}{2} \sigma\left(p p \rightarrow W^{\prime} \rightarrow W Z\right) \lesssim 6-3 \mathrm{fb}, \quad(M=1.8-2 \mathrm{TeV}) .
$$

We have checked that this bound indeed introduces some tension with the expected signal in our model, typically leaving a very narrow allowed strip in the $g_{1}-g_{q}$ plane. However, this bound requires the very strong assumption that the efficiencies for a spin-2 mediator are similar to those of a spin- 1 mediator like the ones present in our model. Thus, we can only take this bound as an indication of the possible constraints of the semileptonic search in the parameter space of our model, and encourage the experimental collaborations to interpret their limits in the context of spin-1 resonances. Neglecting this search, the next most constraining one from the first block of table 1 is the ATLAS $\ell \nu j j$ bound. In our case, we have contribution from both the $W W$ and $Z W$ channels, the former contributing with a factor of 2 due to the multiplicity. Thus, we also impose the following constraint on our signal

$$
2 \sigma\left(p p \rightarrow Z^{\prime} / A^{\prime} \rightarrow W W\right)+\sigma\left(p p \rightarrow W^{\prime} \rightarrow W Z\right) \leq 13-9 \mathrm{fb}, \quad(M=1.8-2 \mathrm{TeV}) .
$$

As we have discussed above, EWPT introduce further constraints in the parameter space of the model. In the case of a universal coupling of light fermions $\left(g_{q}=g_{1}\right)$, the $T$ parameter is proportional to $g_{V}^{2}$ whereas the $S$ parameter is proportional to $g_{q} g_{V}$. Thus, in order to have a set of $\sim 2 \mathrm{TeV}$ vector boson resonances compatible with EWPT we need to have suppressed $g_{V}$ and $g_{q}$. In turn this means a supressed production cross section and supressed branching fraction into dibosons (and therefore less signal for the diboson excess). In the more general case $g_{q} \neq g_{1}$ that we are considering, EWPT can no longer be parameterized in terms of oblique parameters but the tension between EWPT and the generation of our signal is similar. We have implemented EWPT as follows. First we have computed the dimension- 6 effective Lagrangian that results after integrating out the massive vector bosons at tree level. The resulting effective Lagrangian can be read-off from 
eqs. (58)-(66) of [53] with the identifications

$$
\begin{aligned}
& \alpha^{N}=-\frac{g_{V}^{2}}{M^{2}}, \quad \beta_{\psi}^{N}=-\frac{g_{V} g_{\psi}}{M^{2}}, \quad \gamma_{\psi \psi^{\prime}}^{N}=-\frac{g_{\psi} g_{\psi^{\prime}}}{M^{2}}, \\
& \alpha^{D}=\beta_{\psi}^{D}=\gamma_{\psi \psi^{\prime}}^{D}=0 .
\end{aligned}
$$

We have then used the results of [54] to implement the constraints at the $Z$-pole and the ones on [55] to include the constraints on four-fermion interactions. They imply stringent bounds on the parameters of our model (except for $g_{t_{R}}$ ). As an example we show in figure 1 the allowed region in the $g_{1}-g_{q}$ (left) and $g_{V}-g_{q}$ (right) planes for $M=2 \mathrm{TeV}, g_{q_{3}}=0.5$ and different values of the remaining free parameter. As we can see from the figure, there is a very stringent constraint on $g_{V}$

$$
g_{V} \lesssim 1.2(\mathrm{EWPT})
$$

quite independent of the other parameters. Reducing the mass of the vector resonances makes these limits more stringent. We have only included the tree-level contribution of the vector resonances to EWPT. Loop contributions can be very significant in non-custodial composite Higgs models [23] (see also [56-58] for the case of important loop contributions in custodially invariant models). However they are more model-dependent and in particular they cannot be directly correlated to the diboson excess we are trying to explain. Specific realizations of our general parameterization of non-custodial composite Higgs models will have to include such constraints to determine the viability of complete models to explain the observed diboson excess.

\section{Results}

The discussion in the previous section makes it clear in which region of parameter space we can expect composite Higgs models to explain the ATLAS diboson signal. We need a sizeable production cross section of the heavy vector resonances, which implies a sizeable $g_{q}$, and a large decay branching fraction into SM vector bosons, thus the largest possible $g_{V}$. A sizeable production cross section then means that the branching fraction into dileptons and $t \bar{t}$ cannot be too large, otherwise the very stringent bounds on these channels would be violated. Finally, we cannot just increase $g_{q}$ arbitrarily without clashing with dijet or other diboson constraints. As we saw in figure 1, EWPT play a further, somewhat intrincate, role in fixing the allowed region of parameter space that might explain the observed ATLAS excess.

We have performed detailed scans over the parameter space of the model, computing the constraints from EWPT and the corresponding production cross sections in all the relevant channels discussed in the previous sections. These cross sections have been computed with MG5 [59], using a model created with FeynRules [60]. Our results are summarized in figure 2 in which we show the region in the $g_{1}-g_{q}$ that successfully reproduces the ATLAS excess, together with the most relevant constraints. The colored areas correspond to the regions that are forbidden by current constraints. In the case of the $W W / W Z$ signal, this 

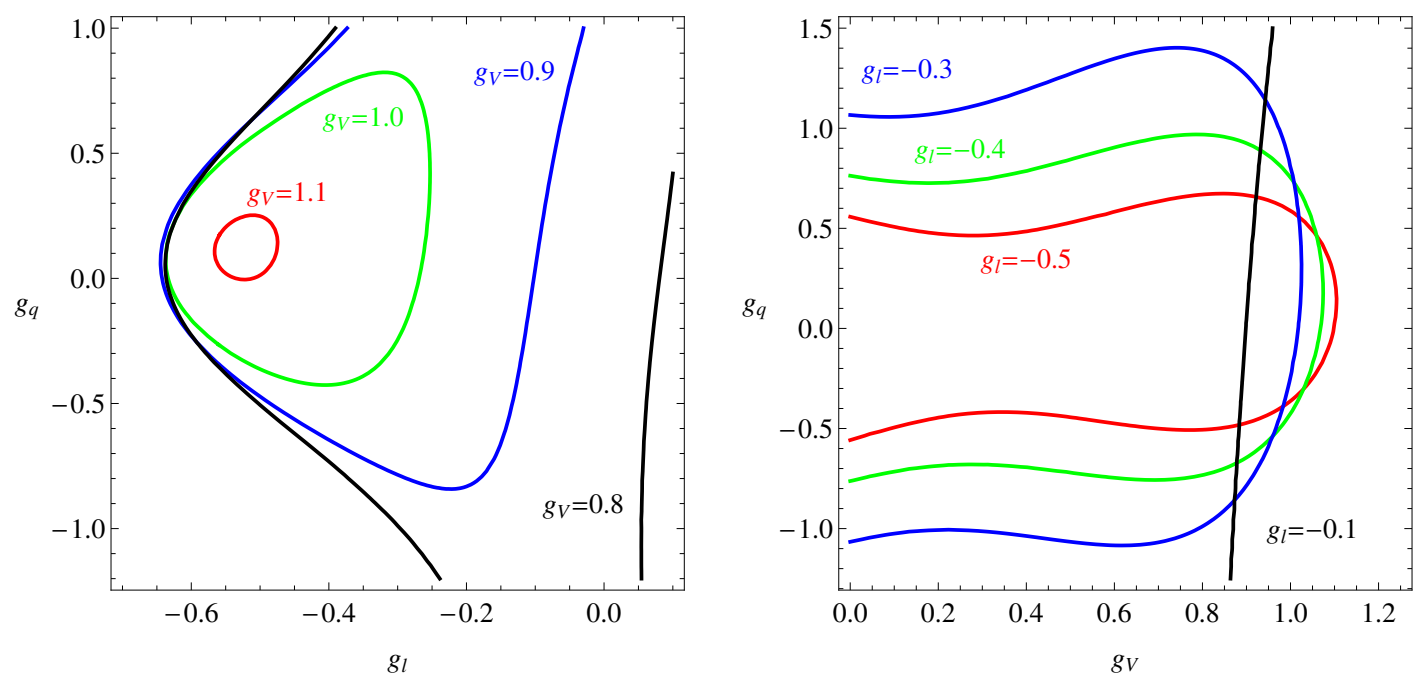

Figure 1. 95\% C.L. allowed regions by EWPT, with $M=2 \mathrm{TeV}$ and $g_{q_{3}}=0.5$, in the $g_{1}-g_{q}$ (left) and $g_{V^{-}} g_{q}$ (right) planes. The allowed regions are the interior of the corresponding contours on the left figure and the region to the left of the contours on the right figure.

means that either the sum of the cross section in both channels is smaller than $5 \mathrm{fb}$ (upper red region) or that the bound in eq. (3.12) is not satisfied (lower red region). All the other bounds are color coded as the label indicates. The white area corresponds to the allowed region that generates the observed signal excess. The input parameters in the figure have been fixed to $M=1.8 \mathrm{TeV}$ and $g_{V}=0.75, M=1.9 \mathrm{TeV}$ and $g_{V}=0.8$ and $M=2 \mathrm{TeV}$ and $g_{V}=0.85$ in the top, middle and bottom rows, respectively. The left (right) column corresponds to $g_{t_{\mathrm{R}}}=0.3(0.5)$ and in all six plots we have fixed $g_{q_{3}}=0.3$.

These plots show that there is a very narrow range in $g_{1}$ that is compatible with EWPT and dilepton bounds. Having enough signal requires sizeable $g_{V}$ and $g_{q}$. Bounds from $t \bar{t}$ production constrain the value of $g_{t_{\mathrm{R}}}$ and $g_{q_{3}}$ (the latter is also constrained by EWPT) to be relatively low. Finally, when the coupling to the third generation quarks are small enough, the upper bound on diboson production in eq. (3.12) and dijet production become the most stringent constraints that prevent the strength of $g_{q}$ to be too large. Combining all these constraints we find the following region in the parameter space for our non-custodial composite Higgs model that can explain the observed diboson excess without contradicting any other experimental bound

$$
g_{V} \sim 0.8-0.9, \quad g_{t_{\mathrm{R}}} \sim g_{q_{3}} \sim 0.3, \quad-0.05 \lesssim g_{1} \lesssim-0.01, \quad-0.5 \lesssim g_{q} \lesssim-0.3
$$

where, depending on the exact values of the parameters (including $M$ ) slightly smaller or larger ranges can be allowed.

Before going to our conclusions let us mention that there are some small excesses in other analyses, like $W H$ or dijets which could also be explained, at least partially, in the same region of parameter space needed to explain the resonant diboson excess. We have not tried to fit any of them but it is worth keeping an eye on any forthcoming analysis. 

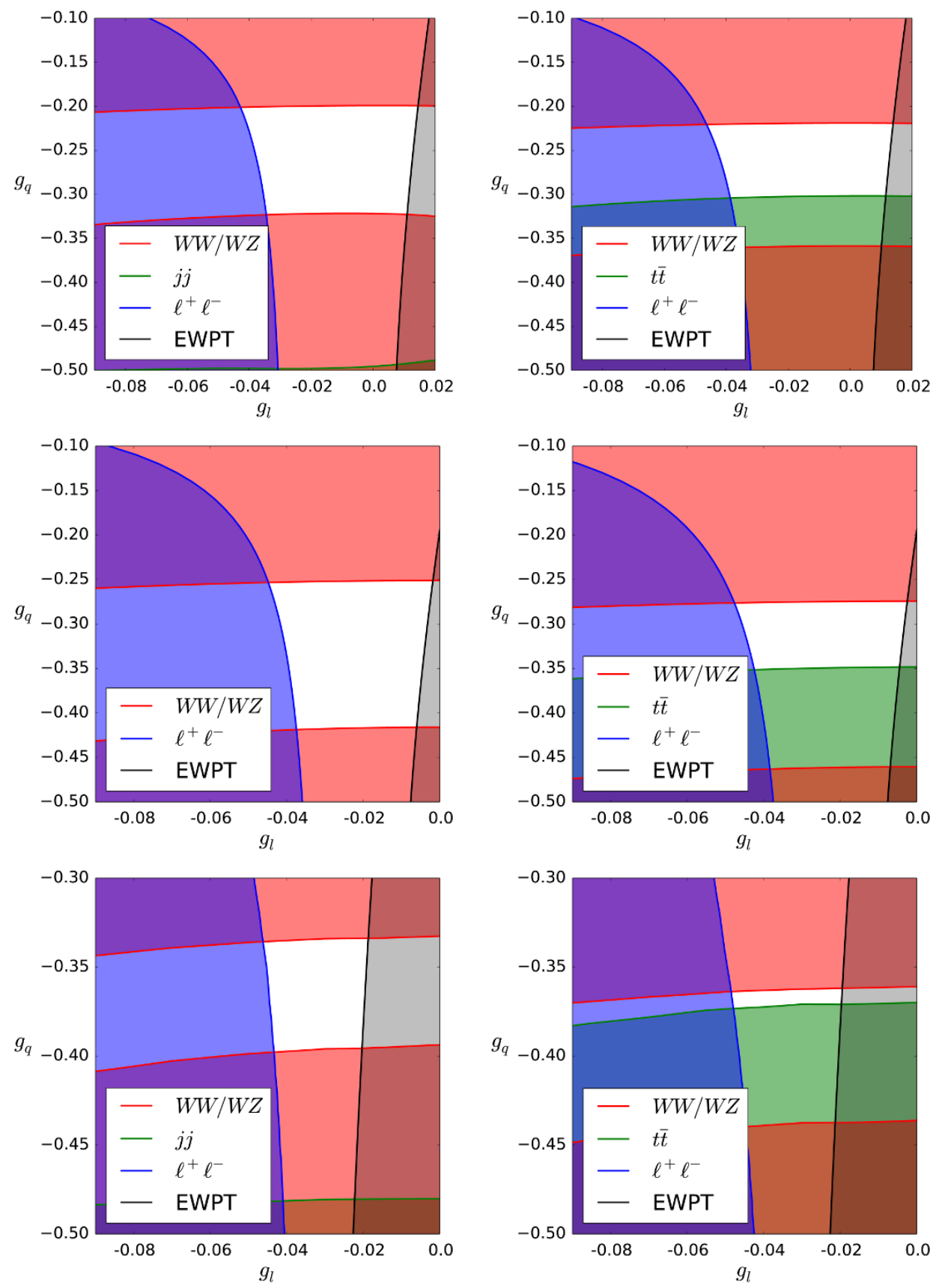

Figure 2. Constraints on the model from the diboson signal, $t \bar{t}, j j, \ell^{+} \ell^{-}$and EWPT. The colored areas correspond to the region excluded by the different bounds. The input parameters in the figure have been fixed to $M=1.8 \mathrm{TeV}$ and $g_{V}=0.75, M=1.9 \mathrm{TeV}$ and $g_{V}=0.8$ and $M=2 \mathrm{TeV}$ and $g_{V}=0.85$ in the top, middle and bottom rows, respectively. The left (right) column corresponds to $g_{t_{\mathrm{R}}}=0.3(0.5)$ and in all six plots we have fixed $g_{q_{3}}=0.3$. 
Any complete UV model will have different correlated predictions in different channels that hopefully the run 2 of the LHC will be able to confirm.

\section{Conclusions}

In this paper we have tried to give an explanation to the recently reported excess in diboson resonant production in the context of non-custodial composite models. Composite Higgs models naturally predict the existence of spin-1 resonances, among other ones, which could explain the observed excess. We have shown that in a particular class of model based on a soft-wall construction one can satisfy all bounds from EWPT and direct searches and still be able to reproduce the resulting observed excess. The mass range reported for the excess, $M \sim 2 \mathrm{TeV}$, is however somewhat low to be accommodated in standard warped models, due to the strong bound coming from the $S$-parameter. This is the reason to resort to softwall models, in which the mixing of these resonances with the SM gauge bosons is reduced in such a way that $\sim 2 \mathrm{TeV}$ vector resonances are compatible with bounds from EWPT. For the sake of simplicity we have considered a minimal non-custodial composite Higgs model. However we have prefered to remain agnostic regarding the underlying gravitational model and we have therefore parameterized with complete generality the relevant couplings. Despite the tension present in the model between the production of a sizeable diboson cross section and direct and indirect constraints we have found that there are regions of parameter space in which the excess can be completely explained without contradicting any other experimental bound. The global analysis, including all the constraints, that we have performed selects a well-defined region of parameter space in non-custodial composite Higgs models in which lepton-quark universality have to be abandoned (due to the bounds from $\ell^{+} \ell^{-}$searches). We also need a sizeable coupling to the light quarks and a reduced coupling to third generation quarks. This would generically correspond to a holographic soft-wall model with a modest volume factor, essentially conformal leptons and with a not-fully composite top quark. The tension between the signal and experimental bounds also means that, if the excess is confirmed during the LHC run 2, it is likely that other related excesses appear with a similar mass scale. In particular, $t \bar{t}$, dijet, $\ell^{+} \ell^{-}$or diboson resonances in other channels (most notably in the semi-leptonic ones) are expected to be easily visible with the new data. A detailed measurement of the corresponding signals would help disentangle the precise region of parameter space that explains all the excesses.

\section{Acknowledgments}

We would like to thank Jorge de Blas and Gustaaf Brooijmans for useful discussions and the École de Physique des Houches for creating an ideal atmosphere where this project was started. AC is supported by the Swiss National Science Foundation under contract SNSF 200021-143781. The work of AD was supported in part by the National Science Foundation under Grant No. PHY-1215979. MQ is partly supported by the Spanish Consolider-Ingenio 2010 Programme CPAN (Grant number CSD2007-00042), by Grant number CICYT-FEDER-FPA2011-25948, by the Severo Ochoa excellence program 
of MINECO under Grant number SO-2012-0234 and by Secretaria d'Universitats i Recerca del Departament d'Economia i Coneixement de la Generalitat de Catalunya under Grant number 2014 SGR 1450. The work of JS has been partially supported by the European Commission (PITN-GA-2012-316704 HIGGSTOOLS), by MINECO under grants (FPA2010-17915 and FPA2013-47836-C3-2-P) and by Junta de Andalucía grants FQM 101 and FQM 6552.

Open Access. This article is distributed under the terms of the Creative Commons Attribution License (CC-BY 4.0), which permits any use, distribution and reproduction in any medium, provided the original author(s) and source are credited.

\section{References}

[1] ATLAS collaboration, Search for high-mass diboson resonances with boson-tagged jets in proton-proton collisions at $\sqrt{s}=8 \mathrm{TeV}$ with the ATLAS detector, arXiv:1506.00962 [INSPIRE].

[2] CMS collaboration, Search for massive resonances in dijet systems containing jets tagged as $W$ or $Z$ boson decays in pp collisions at $\sqrt{s}=8$ TeV, JHEP 08 (2014) 173 [arXiv: 1405.1994] [INSPIRE].

[3] H.S. Fukano, M. Kurachi, S. Matsuzaki, K. Terashi and K. Yamawaki, 2 TeV Walking Technirho at LHC?, arXiv:1506.03751 [INSPIRE].

[4] J. Hisano, N. Nagata and Y. Omura, Interpretations of the ATLAS Diboson Resonances, Phys. Rev. D 92 (2015) 055001 [arXiv:1506.03931] [InSPIRE].

[5] D.B. Franzosi, M.T. Frandsen and F. Sannino, Diboson Signals via Fermi Scale Spin-One States, arXiv: 1506.04392 [INSPIRE].

[6] K. Cheung, W.-Y. Keung, P.-Y. Tseng and T.-C. Yuan, Interpretations of the ATLAS Diboson Anomaly, arXiv: 1506.06064 [InSPIRE].

[7] S.-S. Xue, Vector-like $W^{ \pm}$-boson coupling at TeV and fermion-mass hierarchy (two boson-tagged jets vs four quark jets), arXiv:1506.05994 [INSPIRE].

[8] B.A. Dobrescu and Z. Liu, A $W^{\prime}$ Boson near 2 TeV: Predictions for Run 2 of the LHC, arXiv: 1506.06736 [INSPIRE].

[9] J.A. Aguilar-Saavedra, Triboson interpretations of the ATLAS diboson excess, arXiv:1506.06739 [INSPIRE].

[10] A. Alves, A. Berlin, S. Profumo and F.S. Queiroz, Dirac-Fermionic Dark Matter in U(1) $X$ Models, arXiv:1506.06767 [INSPIRE].

[11] Y. Gao, T. Ghosh, K. Sinha and J.-H. Yu, G221 Interpretations of the Diboson and Wh Excesses, arXiv:1506.07511 [INSPIRE].

[12] A. Thamm, R. Torre and A. Wulzer, A composite Heavy Vector Triplet in the ATLAS di-boson excess, arXiv:1506.08688 [INSPIRE].

[13] J. Brehmer, J. Hewett, J. Kopp, T. Rizzo and J. Tattersall, Symmetry Restored in Dibosons at the LHC?, arXiv:1507.00013 [InSPIRE].

[14] Q.-H. Cao, B. Yan and D.-M. Zhang, Simple Non-Abelian Extensions and Diboson Excesses at the LHC, arXiv: 1507.00268 [INSPIRE]. 
[15] G. Cacciapaglia and M.T. Frandsen, Unitarity implications of diboson resonance in the TeV region for Higgs physics, arXiv:1507.00900 [INSPIRE].

[16] K. Agashe, A. Delgado, M.J. May and R. Sundrum, RS1, custodial isospin and precision tests, JHEP 08 (2003) 050 [hep-ph/0308036] [INSPIRE].

[17] K. Agashe, R. Contino and A. Pomarol, The Minimal composite Higgs model, Nucl. Phys. B 719 (2005) 165 [hep-ph/0412089] [INSPIRE].

[18] M. Carena, E. Ponton, J. Santiago and C.E.M. Wagner, Light Kaluza Klein States in Randall-Sundrum Models with Custodial SU(2), Nucl. Phys. B 759 (2006) 202 [hep-ph/0607106] [INSPIRE].

[19] M. Carena, E. Ponton, J. Santiago and C.E.M. Wagner, Electroweak constraints on warped models with custodial symmetry, Phys. Rev. D 76 (2007) 035006 [hep-ph/0701055] [INSPIRE].

[20] A. Falkowski and M. Pérez-Victoria, Electroweak Breaking on a Soft Wall, JHEP 12 (2008) 107 [arXiv: 0806.1737] [INSPIRE].

[21] J.A. Cabrer, G. von Gersdorff and M. Quirós, Warped Electroweak Breaking Without Custodial Symmetry, Phys. Lett. B 697 (2011) 208 [arXiv:1011.2205] [inSPIRE].

[22] J.A. Cabrer, G. von Gersdorff and M. Quirós, Suppressing Electroweak Precision Observables in 5D Warped Models, JHEP 05 (2011) 083 [arXiv:1103.1388] [INSPIRE].

[23] A. Carmona, E. Ponton and J. Santiago, Phenomenology of Non-Custodial Warped Models, JHEP 10 (2011) 137 [arXiv:1107.1500] [inSPIRE].

[24] J. de Blas, A. Delgado, B. Ostdiek and A. de la Puente, LHC Signals of Non-Custodial Warped 5D Models, Phys. Rev. D 86 (2012) 015028 [arXiv:1206.0699] [InSPIRE].

[25] ATLAS collaboration, Search for production of $W W / W Z$ resonances decaying to a lepton, neutrino and jets in pp collisions at $\sqrt{s}=8 \mathrm{TeV}$ with the ATLAS detector, Eur. Phys. J. C 75 (2015) 209 [Erratum ibid. C 75 (2015) 370] [arXiv: 1503.04677] [INSPIRE].

[26] CMS collaboration, Search for massive resonances decaying into pairs of boosted bosons in semi-leptonic final states at $\sqrt{s}=8 \mathrm{TeV}$, JHEP 08 (2014) 174 [arXiv:1405.3447] [INSPIRE].

[27] ATLAS collaboration, Search for resonant diboson production in the $\ell \ell q \bar{q}$ final state in $p p$ collisions at $\sqrt{s}=8 \mathrm{TeV}$ with the ATLAS detector, Eur. Phys. J. C $7 \mathbf{5}$ (2015) 69 [arXiv: 1409.6190] [INSPIRE].

[28] ATLAS collaboration, Search for $W Z$ resonances in the fully leptonic channel using pp collisions at $\sqrt{s}=8 \mathrm{TeV}$ with the ATLAS detector, Phys. Lett. B 737 (2014) 223 [arXiv: 1406.4456] [INSPIRE].

[29] CMS collaboration, Search for new resonances decaying via WZ to leptons in proton-proton collisions at $\sqrt{s}=8 \mathrm{TeV}$, Phys. Lett. B 740 (2015) 83 [arXiv:1407.3476] [INSPIRE].

[30] ATLAS collaboration, Search for a new resonance decaying to a $W$ or $Z$ boson and a Higgs boson in the $\ell \ell / \ell \nu / \nu \nu+b \bar{b}$ final states with the ATLAS detector, Eur. Phys. J. C 75 (2015) 263 [arXiv: 1503.08089] [INSPIRE].

[31] CMS collaboration, Search for A Massive Resonance Decaying into a Higgs Boson and a $W$ or $Z$ Boson in Hadronic Final States in Proton-Proton Collisions at $\sqrt{s}=8 \mathrm{TeV}$, arXiv: 1506.01443 [INSPIRE]. 
[32] ATLAS collaboration, Search for high-mass dilepton resonances in pp collisions at $\sqrt{s}=8 \mathrm{TeV}$ with the ATLAS detector, Phys. Rev. D 90 (2014) 052005 [arXiv:1405.4123] [INSPIRE].

[33] ATLAS collaboration, Search for new particles in events with one lepton and missing transverse momentum in pp collisions at $\sqrt{s}=8 \mathrm{TeV}$ with the ATLAS detector, JHEP 09 (2014) 037 [arXiv: 1407.7494] [INSPIRE].

[34] CMS collaboration, Search for heavy narrow dilepton resonances in pp collisions at $\sqrt{s}=7 \mathrm{TeV}$ and $\sqrt{s}=8 \mathrm{TeV}$, Phys. Lett. B 720 (2013) 63 [arXiv:1212.6175] [INSPIRE].

[35] CMS collaboration, Search for physics beyond the standard model in final states with a lepton and missing transverse energy in proton-proton collisions at $\sqrt{s}=8 \mathrm{TeV}$, Phys. Rev. D 91 (2015) 092005 [arXiv:1408.2745] [INSPIRE].

[36] ATLAS collaboration, A search for $t \bar{t}$ resonances using lepton-plus-jets events in proton-proton collisions at $\sqrt{s}=8 \mathrm{TeV}$ with the ATLAS detector, JHEP 08 (2015) 148 [arXiv: 1505. 07018] [INSPIRE].

[37] CMS collaboration, Search for Resonant t $\bar{t}$ Production in Proton-Proton Collisions at $\sqrt{s}=8 \mathrm{TeV}$, arXiv:1506.03062 [INSPIRE].

[38] ATLAS collaboration, Search for new phenomena in the dijet mass distribution using pp collision data at $\sqrt{s}=8 \mathrm{TeV}$ with the ATLAS detector, Phys. Rev. D 91 (2015) 052007 [arXiv:1407.1376] [INSPIRE].

[39] CMS collaboration, Search for resonances and quantum black holes using dijet mass spectra in proton-proton collisions at $\sqrt{s}=8 \mathrm{TeV}$, Phys. Rev. D 91 (2015) 052009 [arXiv: 1501.04198] [INSPIRE].

[40] ATLAS collaboration, Search for high-mass diboson resonances with boson-tagged jets in proton-proton collisions at $\sqrt{s}=8 \mathrm{TeV}$ with the ATLAS detector, arXiv:1506.00962 [INSPIRE] and online at https://atlas.web.cern.ch/Atlas/GROUPS/PHYSICS/PAPERS/EXOT-2013-08/.

[41] D.B. Kaplan and H. Georgi, SU(2) $\times$ U(1) Breaking by Vacuum Misalignment, Phys. Lett. B 136 (1984) 183 [INSPIRE].

[42] D.B. Kaplan, H. Georgi and S. Dimopoulos, Composite Higgs Scalars, Phys. Lett. B 136 (1984) 187 [INSPIRE].

[43] R. Contino, Y. Nomura and A. Pomarol, Higgs as a holographic pseudo-Goldstone boson, Nucl. Phys. B 671 (2003) 148 [hep-ph/0306259] [INSPIRE].

[44] R. Barcelo, A. Carmona, M. Chala, M. Masip and J. Santiago, Single Vectorlike Quark Production at the LHC, Nucl. Phys. B 857 (2012) 172 [arXiv:1110.5914] [InSPIRE].

[45] C. Bini, R. Contino and N. Vignaroli, Heavy-light decay topologies as a new strategy to discover a heavy gluon, JHEP 01 (2012) 157 [arXiv:1110.6058] [INSPIRE].

[46] M. Chala, J. Juknevich, G. Perez and J. Santiago, The Elusive Gluon, JHEP 01 (2015) 092 [arXiv: 1411.1771] [INSPIRE].

[47] A. Azatov, D. Chowdhury, D. Ghosh and T.S. Ray, Same sign di-lepton candles of the composite gluons, JHEP 08 (2015) 140 [arXiv: 1505.01506] [INSPIRE].

[48] J. Santiago, Minimal Flavor Protection: A New Flavor Paradigm in Warped Models, JHEP 12 (2008) 046 [arXiv:0806.1230] [INSPIRE]. 
[49] F. del Aguila, A. Carmona and J. Santiago, Neutrino Masses from an $A_{4}$ Symmetry in Holographic Composite Higgs Models, JHEP 08 (2010) 127 [arXiv:1001.5151] [INSPIRE].

[50] J.A. Cabrer, G. von Gersdorff and M. Quirós, Flavor Phenomenology in General 5D Warped Spaces, JHEP 01 (2012) 033 [arXiv:1110.3324] [INSPIRE].

[51] G. von Gersdorff, M. Quirós and M. Wiechers, Neutrino Mixing from Wilson Lines in Warped Space, JHEP 02 (2013) 079 [arXiv: 1208.4300] [INSPIRE].

[52] A. Carmona and F. Goertz, A naturally light Higgs without light Top Partners, JHEP 05 (2015) 002 [arXiv: 1410.8555] [INSPIRE].

[53] H. Davoudiasl, S. Gopalakrishna, E. Ponton and J. Santiago, Warped 5-Dimensional Models: Phenomenological Status and Experimental Prospects, New J. Phys. 12 (2010) 075011 [arXiv:0908.1968] [INSPIRE].

[54] A. Efrati, A. Falkowski and Y. Soreq, Electroweak constraints on flavorful effective theories, JHEP 07 (2015) 018 [arXiv: 1503.07872] [INSPIRE].

[55] J. de Blas, M. Chala and J. Santiago, Global Constraints on Lepton-Quark Contact Interactions, Phys. Rev. D 88 (2013) 095011 [arXiv:1307.5068] [InSPIRE].

[56] C. Anastasiou, E. Furlan and J. Santiago, Realistic Composite Higgs Models, Phys. Rev. D 79 (2009) 075003 [arXiv: 0901.2117] [inSPIRE].

[57] C. Grojean, O. Matsedonskyi and G. Panico, Light top partners and precision physics, JHEP 10 (2013) 160 [arXiv: 1306.4655] [INSPIRE].

[58] R. Contino and M. Salvarezza, One-loop effects from spin-1 resonances in Composite Higgs models, JHEP 07 (2015) 065 [arXiv: 1504.02750] [INSPIRE].

[59] J. Alwall et al., The automated computation of tree-level and next-to-leading order differential cross sections and their matching to parton shower simulations, JHEP 07 (2014) 079 [arXiv: 1405.0301] [INSPIRE].

[60] A. Alloul, N.D. Christensen, C. Degrande, C. Duhr and B. Fuks, FeynRules 2.0 - A complete toolbox for tree-level phenomenology, Comput. Phys. Commun. 185 (2014) 2250 [arXiv: 1310.1921] [INSPIRE]. 